\title{
Increased prostanoid dependency of arterial relaxation in Chlamydia pneumoniae-infected mice
}

\begin{abstract}
Correspondence
Liisa Törmäkangas

liisa.tormakangas@ktl.fi
\end{abstract}

Received 11 January 2006

Accepted 7 April 2006

\author{
Liisa Törmäkangas, ${ }^{1}$ Juha Ketonen, ${ }^{2}$ Maija Leinonen, ${ }^{1}$ Pekka Saikku ${ }^{3}$ \\ and llari Paakkari \\ ${ }^{1}$ National Public Health Institute (KTL), PO Box 310, FIN-90101 Oulu, Finland \\ ${ }^{2}$ Institute of Biomedicine, University of Helsinki, Finland \\ ${ }^{3}$ Department of Medical Microbiology, University of Oulu, Finland
}

\section{INTRODUCTION}

The intracellular, Gram-negative pathogen Chlamydia pneumoniae, which generally causes mild upper respiratory tract infections and occasionally bronchitis and pneumonia in humans worldwide, has been suggested to participate in the development of cardiovascular disease (Leinonen \& Saikku, 2002). In murine models, repeated intranasal inoculations of C. pneumoniae can accelerate atherosclerotic development, especially in hyperlipidaemic mice (Hu et al., 1999; Moazed et al., 1999) and, recently, C. pneumoniae inoculation was also shown to cause aortic endothelial dysfunction in apolipoprotein E-knockout (apoE-ko) mice after repeated C. pneumoniae infections (Liuba et al., 2000) or after a combined infection with Helicobacter pylori (Liuba et al., 2003). This was suggested to be due to a decrease in nitric oxide (NO) availability, caused by the infections, as aortic relaxation in the system was significantly impaired by an inhibitor of nitric oxide synthase (NOS), $N^{\omega}$-nitro-Larginine methyl ester (L-NAME). A possible effect of cyclooxygenases (COXs) and prostanoids was also proposed, but these results were not quite as conclusive (Liuba et al., 2000).

\footnotetext{
Abbreviations: COX, cyclooxygenase; EDHF, endothelium-derived hyperpolarizing factor; GAPDH, glyceraldehyde-3-phosphate dehydrogenase; 6-keto $\mathrm{PGF}_{1 \alpha}$, 6-keto prostaglandin $\mathrm{F}_{1 \alpha} ; \mathrm{MC}$, methacholine; L-NAME, $N^{\omega}$-nitro-L-arginine methyl ester; NO, nitric oxide; NOS, nitric oxide synthase; $\mathrm{PGI}_{2}$, prostaglandin $\mathrm{I}_{2}$.
}

Endothelial relaxation is commonly known to be mediated via $\mathrm{NO}$ and via prostaglandin $\mathrm{I}_{2}\left(\mathrm{PGI}_{2}\right)$ produced by vascular COX but, a third mechanism, an endothelium-derived hyperpolarizing factor (EDHF) that does not respond to NOS or COX inhibitors, could also mediate vascular relaxation, possibly by hyperpolarizing smooth muscle cells through activation of $\mathrm{K}^{+}$channels in both endothelial and smooth muscle cells (Feletou \& Vanhoutte, 1999). However, the chemical identity of EDHF, if any, is not clear.

Endothelial dysfunction plays an important role in the development of atherosclerosis (Ross, 1999). In this study, dysfunction detected as impaired relaxation responses was assessed. Wild-type mice fed a standard chow diet are normocholesterolaemic and do not develop atherosclerotic lesions spontaneously; however, C57BL/6J mice have been shown to be susceptible to atherosclerosis when fed an atherogenic diet (Paigen et al., 1985) and are also vulnerable to changes similar to the human metabolic syndrome (Collins et al., 2004). We have shown previously that repeated C. pneumoniae inoculations can lead to an increase in sub-intimal lipid accumulation in aortic sinuses of normocholesterolaemic C57BL/6J mice fed a diet with a slight cholesterol supplement (Törmäkangas et al., 2005a). Thus, the aim of the present study was to determine whether acute C. pneumoniae infection is able to induce endothelial dysfunction measured as altered relaxation responses in these wild-type C57BL/6J mice. 


\section{METHODS}

Animal model and samples. Inbred C57BL/6J female mice purchased from Harlan Netherlands were divided into two groups of nine animals at the age of 8 weeks. C. pneumoniae isolate Kajaani 7, free from mycoplasma, was used to inoculate the mice intranasally. The first group was inoculated with $7 \times 10^{5}$ inclusion-forming units of C. pneumoniae per mouse in sucrose/phosphate/glutamate (SPG) buffer under inhaled methoxyflurane (Medical Developments Australia) anaesthesia. The second group was inoculated with SPG instead of chlamydia. The mice were sacrificed using $\mathrm{CO}_{2}$ and samples were collected 4 days post-inoculation (p.i.). Chlamydia culture, quantitative $\mathrm{COX}$ analyses and measurement of $\mathrm{PGI}_{2}$ levels were done for each of the nine animals in both groups. The aortas from only six randomly selected mice from each group were taken for vascular reactivity studies because of limited instrumentation. The Animal Care and Use Committee of the National Public Health Institute, Helsinki, Finland approved all procedures involving animals.

Chlamydia culture. Culture from mechanically homogenized lung tissue was performed as described in detail previously (Erkkilä et al., 2002). Pathfinder (Sanofi Diagnostics Pasteur) Chlamydia genusspecific monoclonal antibody conjugated to fluorescein isothiocyanate was used to detect the chlamydia inclusions in HL (a human epithelial cell line) cells.

Detection of chlamydial DNA in aortic tissue. Sections of the aortas collected from the six infected and uninfected mice for vascular reactivity measurements were analysed for the presence of chlamydial DNA in the tissue. Descending aortic tissue was homogenized for Western blot analysis and the supernatant collected after centrifugation of the homogenate was studied by using PCR. Purification of DNA using a commercially available QIAamp tissue kit and a nested-PCR assay combining conventional and LightCycler real-time methods for detecting the C. pneumoniae pst fragment were done as described previously (Törmäkangas et al., 2005a). Pstl is a $C$. pneumoniae-specific genome fraction with no known function at present.

Quantitative mouse COX-1 and COX-2 mRNA analyses. Pieces of about $10-25 \mathrm{mg}$ of the right lung from each of the nine mice per study group were stored in RNAlater RNA Stabilization reagent (Qiagen). Total RNA was extracted and reverse transcription was performed as described elsewhere (Törmäkangas et al., 2005a). A quantitative LightCycler analysis for cDNA was performed with specific primers based on COX-1 and COX-2 mRNA sequences (Tanigawa et al., 2004). The PCR reaction mixtures (final volume $20 \mu \mathrm{l}$ ) consisted of $2 \cdot 5 \mathrm{mmol} \mathrm{MgCl}_{2} \mathrm{l}^{-1}$ for COX-2 and $3 \mathrm{mmol}$ $\mathrm{MgCl}_{2} \mathrm{1}^{-1}$ for COX-1, $0.5 \mathrm{mmol} 1^{-1}$ of each primer, $2 \mu \mathrm{l}$ FastStart DNA Master SYBR Green I mix and $2 \mu \mathrm{l}$ cDNA template. After pre-incubation at $95^{\circ} \mathrm{C}$, the amplification cycles were: COX-1, $95^{\circ} \mathrm{C} /$ $10 \mathrm{~s}, 60^{\circ} \mathrm{C} / 10 \mathrm{~s}$ and $72^{\circ} \mathrm{C} / 5 \mathrm{~s} ; \operatorname{COX}-2,95^{\circ} \mathrm{C} / 15 \mathrm{~s}, 61^{\circ} \mathrm{C} / 10 \mathrm{~s}$ and $72^{\circ} \mathrm{C} / 9 \mathrm{~s}$. Specific standards for all genes were produced by purification of the correct PCR products from agarose gels, amplification of these products with LightCycler and concentration of the amplified products by ethanol precipitation. The amount of the specific sequence in each stock was determined and the stocks were diluted to obtain standards of $1-10^{4}$ genomes $\mu 1^{-1}$. Quantification was done by using a standard curve and calculating the results using the second-derivative maximum method of the LightCycler Data Analysis software (version 3.5.28). A melting curve analysis for each LightCycler run was performed and the expression of a housekeeping gene encoding glyceraldehyde-3-phosphate dehydrogenase (GAPDH) was measured from the cDNA samples, according to the method described by Simpson et al. (2000). The crossing point $\left(C_{\mathrm{p}}\right)$ value for the GAPDH and COX analyses represents the PCR cycle at which the increase in the SYBR Green fluorescence signal above baseline was detected and indicates the level of template concentration in the sample. When the $C_{\mathrm{p}}$ values for GAPDH cDNA were studied, it was shown that the mRNA levels were affected by infection: the mean $C_{\mathrm{p}}$ (SD) for the uninfected group was $25.4(0.99)$ and $24.6(0.99)$ for the infected group. Infection therefore increased the expression of GAPDH, and GAPDH results were not used for the relative quantification analysis. COX cDNA quantification calculated using the LightCycler software with a standard curve was adjusted for the weight of the tissue taken for RNA extraction. The results are presented per $25 \mathrm{mg}$ lung tissue.

6-keto Prostaglandin $\mathbf{F}_{\mathbf{1} \boldsymbol{\alpha}}$ (6-keto $\mathbf{P G F}_{\mathbf{1} \boldsymbol{\alpha}}$ ) levels. The main metabolite of $\mathrm{PGI}_{2}$, 6-keto $\mathrm{PGF}_{1 \alpha}$, in the serum samples was measured by using a commercial enzyme immunoassay kit (no. 515211; Cayman Chemical). The samples were diluted $1: 2$ using the kit EIA buffer and the analysis was performed according to the manufacturer's instructions.

Vascular reactivity studies. Six aortas from both infected and uninfected control mice were analysed for endothelial dysfunction. The fat tissue surrounding the upper section of the descending thoracic aorta was removed and the aorta was cut into rings (approximately $3 \mathrm{~mm}$ wide). The endothelium-intact rings were placed between steel hooks, mounted in an organ bath chamber and equilibrated for $60 \mathrm{~min}$ under a resting tension of $0.7 \mathrm{~g}$ in oxygenated $\left(96 \% \mathrm{O}_{2} / 4 \% \mathrm{CO}_{2}\right)$ Krebs solution $\left(\mathrm{pH} 7 \cdot 4,37^{\circ} \mathrm{C}\right)$ after a first precontraction with $1.2 \mathrm{~g}$. The force of contraction was measured with an isometric force-displacement transducer using a computerized system (EMKA 2000). The presence of intact endothelium in the vascular preparation was confirmed by observing the relaxation response to $1 \mu \mathrm{mol}$ methacholine (MC) $1^{-1}$ (acetyl- $\beta$-methacholine chloride) in rings pre-contracted with $0 \cdot 1 \mu \mathrm{mol}$ noradrenaline $1^{-1}$ (bitartrate salt). To measure the endothelial function, the aortic rings were relaxed with increasing concentrations of MC after noradrenaline contraction. In addition, the effects of the nonselective NOS enzyme inhibitor L-NAME (at a concentration of $10^{-4} \mathrm{~mol} \mathrm{l}^{-1}$ ) and the non-selective COX inhibitor diclofenac (diclofenac sodium salt; at a concentration of $10^{-6} \mathrm{~mol} \mathrm{l}^{-1}$ ) on arterial relaxation were measured by incubating the aortic rings in these solutions in organ baths prior to relaxation assays. Noradrenaline, MC, L-NAME and diclofenac were obtained from Sigma-Aldrich.

Statistical analysis. The endothelial relaxation responses of the groups were compared using 2-way ANOVA for repeated measures. Differences between the groups after quantitative COX-expression analyses were tested with the non-parametric Mann-Whitney U test. Statistics were done using SPSS version 11.5.1.

\section{RESULTS AND DISCUSSION}

In this study, we analysed the effect of acute C. pneumoniae infection on aortic endothelial function in a C57BL/6J mouse model. Eight of nine mice were found to be culturepositive, with high inclusion numbers from lung tissue, thus proving that the inoculation was successful. The one mouse that was negative was excluded from further analyses and was not among the six animals studied for vascular reactivity. None of the SPG-inoculated control mice were positive. Aortic tissue from the six mice from each group that were studied for aortic relaxation was analysed for the presence of chlamydial DNA. Four of the six aortas from the group infected with chlamydia were found to be positive with the nested-PCR method used, thus indicating the presence of chlamydia in the aortic tissue samples, whereas all six aortas from the uninfected mice analysed in the same 
PCR were negative. Although the nested-PCR method used in these analyses is not quantitative, it indicated that the genome numbers in all four positive samples were very low, thus increasing the possibility of false-negative results. Because of this and the fact that only a very small section of aortic tissue was taken for DNA extraction, the negative PCR result from two infected mice does not prove that these aortas were not infected. As these animals showed successful infection detected by culture, they were not excluded from the vascular reactivity analyses. The PCR analysis of the aortic tissue was done merely to show that chlamydia are able to invade the tissue.

The inoculum dose and C. pneumoniae isolate used in this study have been shown in our previous studies to induce histologically severe, but self-restricted, pneumonitis in C57BL/6J mice (Törmäkangas et al., 2005b), leading to the systemic spread of the pathogen into the vasculature and increased aortic sinus lipid accumulation (Erkkilä et al., 2004; Törmäkangas et al., 2005a). The infectious dose used does not cause serious clinical symptoms or deaths in this mouse model.

\section{Quantification of COX mRNA expression in lung tissue}

The infected group showed an increase in COX-2 expression levels compared with the uninfected group, which was close to significant $(P=0 \cdot 074$; Fig. 1b). No significant differences in the expression levels of COX-1 were detected (Fig. 1a). Infection appeared to increase the expression of the inducible form of cyclooxygenase, COX-2, in lung tissue in our study; however, this finding could not be correlated with the aortic relaxation results. Further studies should also assess the mRNA and protein expression of COXs in aortic tissue. A previous in vitro study showed an increase in COX-2

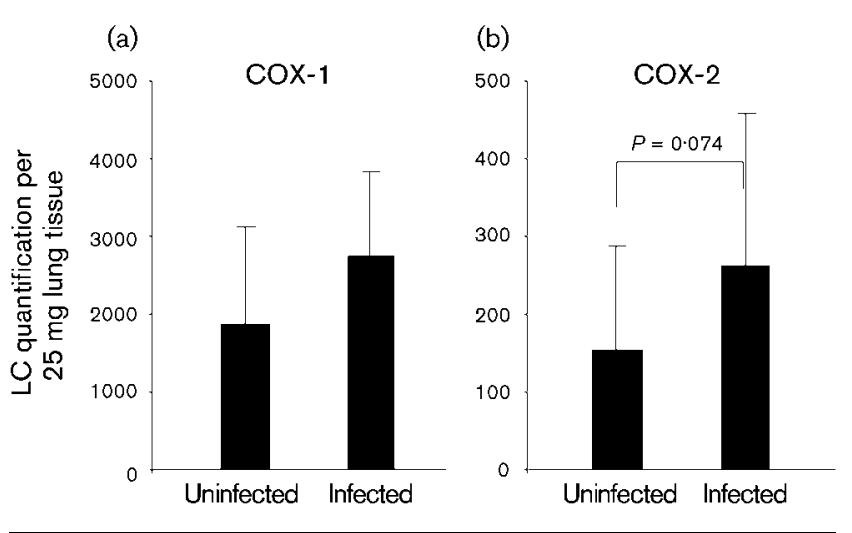

Fig. 1. Expression levels of COX-1 (a) and COX-2 (b) mRNA in mouse lung tissue. Bars represent geometric means and $95 \%$ upper confidence interval. The quantification results were adjusted for the weight of the tissue taken for mRNA extraction. LC, LightCycler. expression that was sustained at a lower level after the rapid induction by $C$. pneumoniae infection in human peripheral blood mononuclear cells (Rupp et al., 2004). The results here show the situation 4 days after infection and it is possible that the peak of the response also occurs soon after primary infection in vivo. $\mathrm{PGI}_{2}$ is produced mainly by vascular endothelium and its metabolite 6-keto $\mathrm{PGF}_{1 \alpha}$ is commonly used to estimate $\mathrm{PGI}_{2}$ synthesis. Production of $\mathrm{PGI}_{2}$ is increased in response to an inflammatory stimulus via increased COX-2 expression, and the function of this prostanoid is to induce vasodilatation and inhibition of platelet aggregation. The slight increase in COX-2 levels detected did not predict the presence of increased 6-keto $\mathrm{PGF}_{1 \alpha}$ levels in the serum samples in this study; no difference was detected in the metabolite concentrations between infected and uninfected mice (results not shown). This finding does not support, but is not able to contradict, the results presented below showing that COX-mediated vasodilatation is increased in the aortas of the infected mice. COX-mediated vasodilatation can also be affected by other prostanoids, in particular prostaglandin $\mathrm{E}_{2}$, which is the major prostanoid produced by COX-2 in response to inflammation.

\section{Endothelial function}

The effects of inoculation on aortic endothelial function are shown in Fig. 2. The aortas from both study groups relaxed similarly in response to increasing MC concentrations when no pre-treatments were used (Fig. 2a). Only a non-significant decrease of 5-10\%, depending on the MC concentration, was seen in the aortas from infected control mice compared with the uninfected group. In the uninfected group, the MC-induced relaxation was mainly $\mathrm{NO}$ dependent, as shown by the inhibitory effect of L-NAME (Fig. 2b) and the lack of a similar effect of diclofenac (Fig. 2c). In the presence of infection, relaxation was dependent on NOS products (presumably NO), but also significantly on COX products (presumably prostanoids), as the relaxation effects of $\mathrm{MC}$ were antagonized by both L-NAME and diclofenac (Fig. 2b, c) and were completely abolished by their combination (Fig. 2d). The difference between the uninfected and infected groups was significant after diclofenac pre-treatment $(P=0 \cdot 001$; relaxation with the highest MC concentration used was about 90 vs $60 \%$, respectively; Fig. 2c) and after combined pre-treatments with both inhibitors $(P=0.031$; Fig. $2 \mathrm{~d})$, but not after L-NAME pre-treatment $(P=0 \cdot 17$; Fig. $2 \mathrm{~b})$.

Although infection did not impair aortic relaxation, it clearly shifted the balance of endothelium-derived relaxing factors from $\mathrm{NO}$ towards vasorelaxing prostanoids. This was shown by pre-treatments with L-NAME and diclofenac. In non-infected aortas, MC-induced relaxation was predominantly NO-dependent (blocked greatly by the NOS inhibitor), whereas the blocking of prostanoid production by the COX inhibitor did not modify the relaxations. However, in vessels from infected mice, the relaxations were still strongly inhibited by L-NAME but the relaxation response was not 

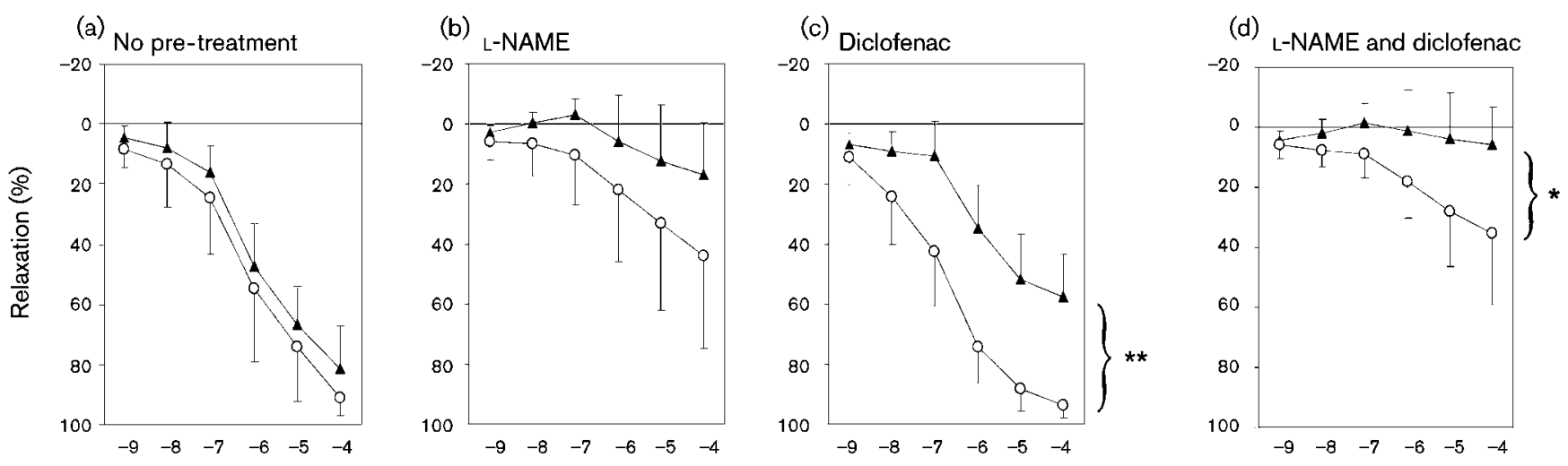

$\log \left(\right.$ methacholine concn, $\left.\mathrm{mol} \Gamma^{-1}\right)$

Fig. 2. Aortic relaxation responses to increasing $M C$ concentrations. (a) Relaxation without pre-treatment, (b) relaxation after L-NAME pre-treatment, (c) relaxation after diclofenac pre-treatment and (d) relaxation after both L-NAME and diclofenac pretreatments. $\bigcirc$, Uninfected control group; $\boldsymbol{\Lambda}$, infected group. Values are means \pm SD for the six mice. ${ }^{\star} P<0 \cdot 05 ;{ }^{\star \star} P<0 \cdot 01$.

significantly different when compared with the uninfected group, whereas diclofenac strongly and significantly impaired relaxation in the infected group. The contribution of both NOS- and COX-mediated relaxation in the infected group was shown by the almost total absence of any vasorelaxing activity of MC after combined blocking of both NOS and COX. In addition to these responses, the presence of another endothelial relaxing factor, possibly EDHF, in the normal uninfected aortas only could be suggested by the present results. A statistically significant relaxation in the presence of the combined blocking of both NOS and COX was observed in the uninfected group, but not in the chlamydia-infected group. However, the potential role of EDHF in this relaxation response should be verified in further studies using the NOS inhibitor and NO scavenger together with diclofenac, to exclude possible incomplete blocking of NOS.

The effects of C. pneumoniae infection on the aortic endothelial function of the apoE-ko mouse model have been studied previously (Liuba et al., 2000). The effects of L-NAME in the present study were similar to those reported by Liuba et al. (2000) after primary inoculation, but the results from diclofenac pre-treatment are partly contradictory. In both studies, COX inhibition had no effect on uninfected mice, but Liuba et al. (2000) suggested that vasoconstricting prostanoids were present after both primary and repeated inoculations in the aortas of apoE-ko mice. In our study of C57BL/6J mice, prostanoid-mediated vasodilatation could be proposed in the infected animals. Use of a different mouse strain may explain the partial differences between our results and those reported by Liuba et al. (2000). In contrast to wild-type C57BL mice, apoE-ko mice are hypercholesterolaemic and spontaneously develop atherosclerotic changes comparable with human lesions in the aorta. Recent studies also suggest that the inflammatory responses to infection are altered in these animals (Laskowitz et al., 2000).

Blessing et al. (2000) reported that three repeated inoculations are able to cause signs of inflammation in the aortic sinuses of C57BL/6J mice, whereas acute infection alone had no such effect. Also, prolonged persistence of chlamydia in the aortic and other tissues has been reported only after repeated C. pneumoniae inoculations in hyperlipidaemic mice (Moazed et al., 1997). However, the present study shows that transient aortic changes are induced by acute intranasal C. pneumoniae infection in $\mathrm{C} 57 \mathrm{BL} / 6 \mathrm{~J}$ mice. Alterations in the aortic relaxation responses seen on day four after primary inoculation in this study were not detected on day 10 p.i. (results not shown). This is in accordance with results reported previously, in which acute C. pneumoniae inoculation did not lead to aortic endothelial dysfunction at 2 weeks p.i. in apoE-ko mice (Liuba et al., 2000).

In conclusion, C. pneumoniae infection or multiple infections are able to affect and change the endotheliumdependent relaxation responses of aortic tissue in mice, as also shown in a previous report (Liuba et al., 2000). In the present study, the mode of endothelium-derived relaxation was shifted from NO dependency towards prostanoid dependency and, in addition, the relaxation effect of a still unknown agent was abolished. Thus, although $C$. pneumoniae infection may not always impair endotheliummediated relaxation, which was tested here with cholinergic stimulation, it may alter the balance of relaxing factors and even abolish some of their activity. This preliminary study warrants a thorough analysis of the effects of both acute and repeated inoculations on endothelial relaxation mediated by NOS, COX and smooth muscle cell-related pathways, measured at different time-points p.i. in this animal model. The results also call for further studies to answer two questions: 
by which mechanisms is endothelial function altered in different disease states, and is the recently reported prothrombotic property of the selective COX-2 inhibitor, rofecoxib, also associated with adverse changes in vascular endothelial contractility.

\section{ACKNOWLEDGEMENTS}

We thank Aini Bloigu for advice and assistance with the statistical analyses.

\section{REFERENCES}

Blessing, E., Lin, T.-M., Campbell, L. A., Rosenfeld, M. E., Lloyd, D. \& Kuo, C. (2000). Chlamydia pneumoniae induces inflammatory changes in the heart and aorta of normocholesterolemic C57BL/ 6J mice. Infect Immun 68, 4765-4768.

Collins, S., Martin, T. L., Surwit, R. S. \& Robidoux, J. (2004). Genetic vulnerability to diet-induced obesity in the C57BL/6J mouse: physiological and molecular characteristics. Physiol Behav 81, 243-248.

Erkkilä, L., Laitinen, K., Laurila, A., Saikku, P. \& Leinonen, M. (2002). Experimental Chlamydia pneumoniae infection in NIH/S mice: effect of reinoculation with chlamydial or cell preparation on culture, PCR and histological findings of lung tissue. Vaccine 20, 2318-2324.

Erkkilä, L., Laitinen, K., Haasio, K., Tiirola, T., Jauhiainen, M., Lehr, H. A., Aalto-Setälä, K., Saikku, P. \& Leinonen, M. (2004). Heat shock protein 60 autoimmunity and early lipid lesions in cholesterol-fed C57BL/6JBom mice during Chlamydia pneumoniae infection. Atherosclerosis 177, 321-328.

Feletou, M. \& Vanhoutte, P. M. (1999). The third pathway: endothelium-dependent hyperpolarization. J Physiol Pharmacol 50, 525-534.

Hu, H., Pierce, G. N. \& Zhong, G. (1999). The atherogenic effects of chlamydia are dependent on serum cholesterol and specific to Chlamydia pneumoniae. J Clin Invest 103, 747-753.

Laskowitz, D. T., Lee, D. M., Schmechel, D. \& Staats, H. F. (2000). Altered immune responses in apolipoprotein E-deficient mice. J Lipid Res 41, 613-620.

Leinonen, M. \& Saikku, P. (2002). Evidence for infectious agents in cardiovascular disease and atherosclerosis. Lancet Infect Dis 2, 11-17.
Liuba, P., Karnani, P., Pesonen, E., Paakkari, I., Forslid, A., Johansson, L., Persson, K., Wadström, T. \& Laurini, R. (2000). Endothelial dysfunction after repeated Chlamydia pneumoniae infection in apolipoprotein E-knockout mice. Circulation 102, 1039-1044.

Liuba, P., Pesonen, E., Paakkari, I. \& 8 other authors (2003). Co-infection with Chlamydia pneumoniae and Helicobacter pylori results in vascular endothelial dysfunction and enhanced VCAM-1 expression in apoE-knockout mice. J Vasc Res 40, 115-122.

Moazed, T. C., Kuo, C., Grayston, J. T. \& Campbell, L. A. (1997). Murine models of Chlamydia pneumoniae infection and atherosclerosis. J Infect Dis 175, 883-890.

Moazed, T. C., Campbell, L. A., Rosenfeld, M. E., Grayston, J. T. \& Kuo, C. C. (1999). Chlamydia pneumoniae infection accelerates the progression of atherosclerosis in apolipoprotein E-deficient mice. $J$ Infect Dis 180, 238-241.

Paigen, B., Morrow, A., Brandon, C., Mitchell, D. \& Holmes, P. (1985). Variation in susceptibility to atherosclerosis among inbred strains of mice. Atherosclerosis 57, 65-73.

Ross, R. (1999). Atherosclerosis - an inflammatory disease. N Engl $J$ Med 340, 115-126.

Rupp, J., Berger, M., Reiling, N., Gieffers, J., Lindschau, C., Haller, H., Dalhoff, K. \& Maass, M. (2004). Cox-2 inhibition abrogates Chlamydia pneumoniae-induced $\mathrm{PGE}_{2}$ and MMP-1 expression. Biochem Biophys Res Commun 320, 738-744.

Simpson, D. A. C., Feeney, S., Boyle, C. \& Stitt, A. W. (2000). Retinal VEGF mRNA measured by SYBR green I fluorescence: a versatile approach to quantitative PCR. Mol Vis 6, 178-183.

Tanigawa, T., Watanabe, T., Hamaguchi, M. \& 7 other authors (2004). Anti-inflammatory effect of two isoforms of COX in $H$. pylori-induced gastritis in mice: possible involvement of $\mathrm{PGE}_{2} . \mathrm{Am}$ J Physiol Gastrointest Liver Physiol 286, G148-G156.

Törmäkangas, L., Erkkilä, L., Korhonen, T., Tiirola, T., Bloigu, A., Saikku, P. \& Leinonen, M. (2005a). Effects of repeated Chlamydia pneumoniae inoculations on aortic lipid accumulation and inflammatory response in C57BL/6J mice. Infect Immun 73, 6458-6466.

Törmäkangas, L., Vuorela, P., Saario, E., Leinonen, M., Saikku, P. \& Vuorela, H. (2005b). In vivo treatment of acute Chlamydia pneumoniae infection with the flavonoids quercetin and luteolin and an alkyl gallate, octyl gallate, in a mouse model. Biochem Pharmacol 70, 1222-1230. 